\title{
A review of structural magnetic resonance neuroimaging
}

\author{
M Symms, H R Jäger, K Schmierer, T A Yousry
}

J Neurol Neurosurg Psychiatry 2004;75:1235-1244. doi: 10.1136/jnnp.2003.032714

Magnetic resonance imaging (MRI) is often divided into structural MRI and functional MRI (FMRI). The former is a widely used imaging technique in research as well as in clinical practice. This review describes the more important developments in structural MRI in recent years, including high resolution imaging, T2 relaxation measurement, $\mathrm{T}^{*}$-weighted imaging, $\mathrm{T} 1$ relaxation measurement, magnetisation transfer imaging, and diffusion imaging. The principles underlying these techniques, as well as their use in research and in clinical practice, will be discussed. shift imaging aim to measure chemical concentrations, and these should be considered separately from other MR techniques. Note that spectroscopy is usually structural/static in nature, though some functional spectroscopy studies have been done.

It is obviously beyond the scope of any article to assess the whole range of techniques and applications of structural MRI. This review will cover some of the new developments in structural MRI and discuss their principles with examples of their role in research and clinical practice.

\section{APPLICATIONS OF STRUCTURAL MRI}

T2- and T1-weighted sequences form the core of almost every clinical MRI protocol. Pathological processes are therefore most often described in terms of T1 and T2 signal behaviour, in addition to contrast enhancement, anatomical location, and morphological characteristics.

More recently, fluid attenuated inversion recovery (FLAIR) has been introduced as a complement of, or even a replacement for, the conventional T2-weighted sequence. Over the past years new techniques have been developed to improve spatial resolution (high resolution imaging) and sensitivity (T2* imaging), establish quantitative methods (T1 maps, T2 maps, magnetisation transfer imaging (MTI)), and develop new qualitative and quantitative contrast techniques (diffusion imaging). ${ }^{78}$

\section{High resolution imaging \\ Principle}

The two main characteristics which govern image quality are spatial resolution and signal to noise ratio (SNR). In-plane resolution is chiefly determined by the number of picture elements (pixels) in the frequency and phase encoding directions, and through-plane resolution by the slice thickness. SNR is determined by pixel size, slice thickness, scan time (including the number of phase encoding steps) and the sequence used. Image quality is therefore ultimately determined by scan time, which, in turn, is influenced chiefly by patients ability to comply with keeping still.

Abbreviations: $A D C$, apparent diffusion coefficient; $A D$, Alzheimer's disease; CSF, cerebrospinal fluid; CT, computed tomography; DTI, diffusion tensor imaging; DWI, diffusion-weighted imaging; FLAIR, fluid attenuated inversion recovery; fMRI, functional magnetic resonance imaging; $\mathrm{Gd}$, gadolinium; GRE, gradient echo; $M D$, mean diffusivity; MS, multiple sclerosis; MTI,

magnetisation transfer imaging; MTR, magnetisation transfer ratio; NMR, nuclear magnetic resonance; PP/

SPMS, primary progressive/secondary progressive MS; $R F$, radiofrequency; RRMS, relapsing remitting MS; SNR, signal to noise ratio 
Any motion occurring during the MRI scan can cause motion artefacts. Cooperative subjects can be persuaded to keep their head still, and head restraints can minimise head motion, but there are other sources of movement. The regular brain pulsation caused by the cardiac cycle can be mostly averaged out over a long scan or explicitly removed by cardiac triggering. The effects of the respiratory cycle are small in most neuroimaging experiments, but random motions such as eye movement cannot be removed.

\section{Applications}

Research

Coronal Tl-weighted, three dimensional, high resolution images are used to measure the volume of the hippocampus, usually by means of manually tracing its outline. They are also the basis for many cross-sectional and longitudinal studies determining the volume of, and assessing changes in, the hippocampus over time in hippocampal sclerosis and Alzheimer's disease $(\mathrm{AD}){ }^{{ }^{9}}$

\section{Clinical environment}

\section{- Epilepsy}

High resolution Tl-weighted images have become a standard tool in epilepsy. The ability to identify the hippocampus and its structure is paramount for assessing atrophy in hippocampal sclerosis. An example is shown in fig l. Furthermore, accurate visualisation of the cortex is important for the diagnosis of cortical dysplasia.

\section{- Cranial nerves}

Identification of subtle structures within the CSF is facilitated by using sequences with a cisternographic effect. These sequences (3D CISS, DRIVE, FIESTA) enable the reliable identification of fine structures such as the anterior choroidal artery, the abducens nerve and even the trochlear nerve. ${ }^{10}{ }^{11}$ Visualisation of the trochlear nerve has helped to elucidate the pathogenesis of "superior oblique myokymia" by revealing the presence of an arterial neurovascular contact at the root exit zone of the nerve. ${ }^{12}$

High-resolution imaging is the "purest" form of structural imaging, and the need to see the brain's structures in

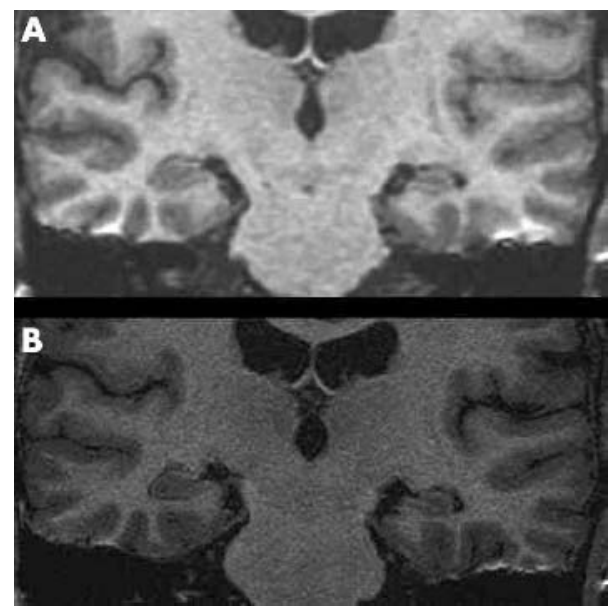

Figure 1 Coronal section of a $\mathrm{Tl}$-weighted three dimensional volume of a subject with epilepsy at (A) conventional $(1 \mathrm{~mm} \times 1 \mathrm{~mm} \times 1.5 \mathrm{~mm})$ and (B) high $(0.5 \mathrm{~mm} \times 0.5 \mathrm{~mm} \times 1 \mathrm{~mm})$ resolution. Hippocampal sclerosis can be seen as the loss of volume in the left hippocampus (right side of image). evermore detail provides the ongoing impetus to increase MRI resolution.

\section{T2 relaxation measurement \\ Principle}

$\mathrm{T} 2$-weighted images are commonly used in neuroimaging because they are very sensitive to a wide range of pathology. However, the signal in $\mathrm{T} 2$-weighted images contains not only $\mathrm{T} 2$ weighting, but also information about the amount of water (proton density) in each pixel. While this extra information can sometimes aid diagnosis, it can also confound it. Using two or more images with different echo times, or by curve-fitting to a long spin echo train, it is possible to calculate $\mathrm{T} 2$ maps of the brain that are independent of proton density.

\section{Applications in research}

Hippocampal sclerosis can be detected in patients with epilepsy by comparing the hippocampal T2 values with a range of values of normal controls..$^{13} \mathrm{~T} 2$-weighted images of a subject with left hippocampal sclerosis are shown in fig 2 .

\section{T2*-weighted imaging \\ Principle}

T2-weighted imaging usually uses images derived from a spin echo, because this refocuses any field inhomogeneities that are present-these are usually caused by changes in magnetic susceptibility between neighbouring tissues. If gradient echo (GRE) images are acquired instead, they will reflect the effects of field inhomogeneity and provide additional contrast: $\mathrm{T} 2{ }^{*}$ weighting.

\section{Applications}

Research

Using modified $\mathrm{T}^{*}$-weighted sequences, it is possible to examine relative levels of iron concentration in the brain: iron content has been observed to be elevated in the substantia nigra in patients with Parkinson's disease. ${ }^{14}$

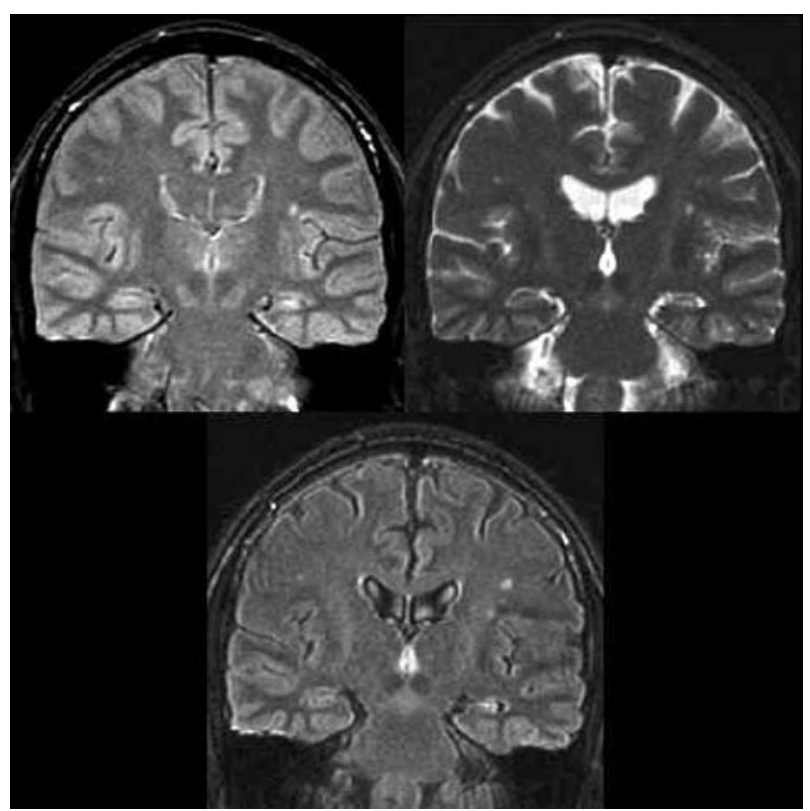

Figure 2 Proton density, T2-weighted and fast FLAIR images of a subject with left hippocampal sclerosis. The left hippocampus (right side of image) has a brighter T2-weighted signal, which is particularly evident on the fast FLAIR image where the surrounding cerebrospinal fluid has been suppressed. 
Clinical environment

\section{- Acute cerebral haemorrhage}

Reliable identification of acute haemorrhage is important for differentiation between a haemorrhagic and an ischaemic stroke, especially if thrombolytic treatment is being considered. Routine spin echo MR images are less sensitive than computer tomography (CT) for detection of acute brain haemorrhage, which is one of the reasons why CT scanning is currently the first choice of imaging modality for acute stroke in clinical practice. However, the detection of acute parenchymal haemorrhage on MRI can be considerably improved by T2*-weighted GRE imaging, which exploits the magnetic susceptibility artefact from deoxyhaemoglobin. Sensitivity of MRI protocols that include $\mathrm{T}^{*}$-weighted GRE images to acute brain haemorrhage has been shown to be similar to that of CT. ${ }^{15} \mathrm{~T} 2$-weighted images associated with a diffusionweighted imaging (DWI) sequence are prone to susceptibility artefacts ${ }^{16}$ - it was therefore suggested to replace the $\mathrm{T}^{*}$ weighted GRE images thereby saving examination time. However, T2*- weighted GRE images have been found to be more sensitive to haemorrhage than the DWI associated T2weighted images and should therefore be an integral part of an MRI protocol for acute stroke. ${ }^{16}$

\section{- Subarachnoid haemorrhage}

CT scanning and lumbar puncture are the current reference standards for detection of subarachnoid haemorrhage. T2* weighted GRE has been shown to be the most useful MRI sequence with $94 \%$ sensitivity for acute subarachnoid haemorrhage and $100 \%$ sensitivity for subacute subarachnoid haemorrhage (after four days). ${ }^{17}$ This imaging modality can therefore be of importance in the delayed diagnosis of subarachnoid haemorrhage, which is difficult with CT.

\section{- Cerebral microhaemorrhages}

Small hypointense areas on $\mathrm{T} 2$ and $\mathrm{T} 2{ }^{*}$-weighted images correspond histopathologically to extravasation of blood and have been dubbed "microhaemorrhages". ${ }^{18}$ These lesions are much more conspicuous on $\mathrm{T}^{*}$-weighted GRE images than on T2-weighted spin echo images due to the increased effects of magnetic susceptibility from haemosiderin deposits. Microhaemorrhages are a feature of hypertensive small vessel disease but have also been described in cerebral amyloid angiopathy, ${ }^{19}$ cerebral autosomal dominant arteriopathy with subcortical infarcts, and leukoencephalopathy $(\text { CADASIL })^{20}$ and trauma.

On T2*-weighted GRE images, microhaemorrhages occurred in $6.4 \%$ of a randomly selected elderly population without neurological disease ${ }^{21}$ and $3.7 \%$ of patients without previous strokes but in $18.1 \%$ of patients with a history of ischaemic stroke and in $71 \%$ of patients with a history of haemorrhagic stroke. ${ }^{22}$ The strong correlation between the presence of microhaemorrhages and a history of haemorrhagic stroke suggests that microhaemorrhages are not only a direct marker of bleeding-prone small vessel disease but could also be a predictor of further haemorrhagic stroke. Examples of the appearance of microhaemorrhages on $\mathrm{T}^{*}$ weighted images are shown in fig 3.

Furthermore, in trauma, microhaemorrhages are a histological hallmark of diffuse axonal injury, and are therefore important to detect. It is interesting to note that the $\mathrm{T} 2$ * lesion load has been found to correlate with the period of loss of consciousness and Glasgow Outcome Scale. ${ }^{23}$ The most sensitive way to detect these microbleeds is to use $\mathrm{T} 2{ }^{*}$ weighted sequences as they detect three times as many lesions as the standard MRI sequences at $1.5 \mathrm{~T}$ and at $3 \mathrm{~T}^{23}$

\section{T1 relaxation measurement}

Principle

T1 mapping follows a rationale similar to T2 mappingremoves variations in image intensity due to proton density by generating an image depending on just one parameter. Tl maps can be generated from two or more images with different repetition times or flip angles, and so have different Tl weightings, or from two or more images with different inversion times, which again have different $\mathrm{Tl}$ weightings. $\mathrm{Tl}$ mapping is much more susceptible than T2 mapping to inhomogeneities of the applied radiofrequency (RF) field, so it is advisable to acquire extra images to measure these imperfections and correct for them. ${ }^{24}$ This is rarely possible at present in the clinical setting.

\section{Applications in research}

In patients with multiple sclerosis (MS) the Tl of normal appearing white matter is significantly different between the infratentorial and supratentorial regions. ${ }^{25}$ This has important consequences for the optimisation of $\mathrm{Tl}$ weighting of sequences used for detecting lesions in MS; optimal and consistent lesion detection may require different $\mathrm{Tl}$ weightings at different levels of the brain.

\section{Magnetisation transfer imaging \\ Principle}

The signal in most MR images arises from the protons in the "mobile pool" - that is, from water in the liquid phase in the body. However, many protons in relatively solid phases are bound in proteins and large biomolecules (the "bound pool") and have a very broad NMR resonance that normally decays too quickly for the MR scanner to detect. These bound protons are in a state of chemical exchange and diffusion with protons in the mobile pool, providing a link between the two pools of protons. If a strong RF pulse is applied which is far enough away from the resonance of the liquid pool, but still excites the bound pool, some of the excited magnetisation is transferred from the bound pool to the liquid pool. The resulting magnetisation transfer (MT)-weighted image is attenuated, and the magnetisation transfer ratio (MTR) can be calculated from this image and a similar one without MT weighting. While MTR is difficult to relate to a real physical measure and MTR measures vary according to the imaging sequence and scanner used, it has nevertheless proved to be a sensitive marker of pathological change in many neurological
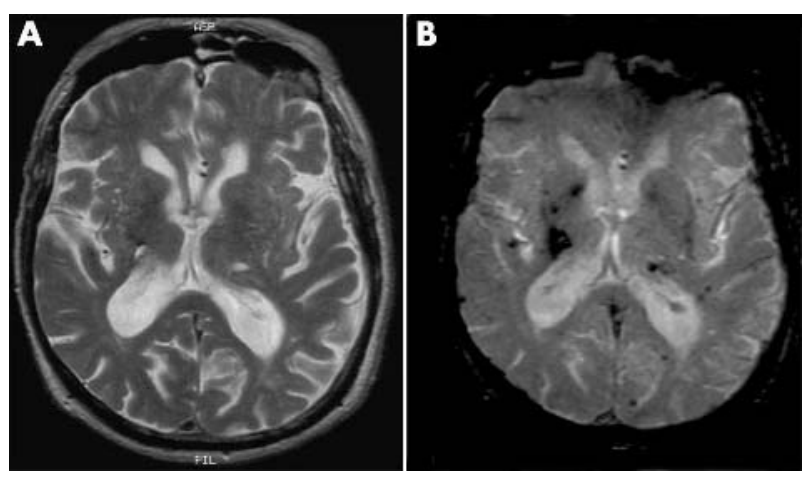

Figure 3 (A) T2-weighted fast spin echo (FSE) and (B) T2*-weighted gradient echo (GRE) images of a 66 year old patient with an old right basal ganglia haemorrhage and extensive cerebral microhaemorrhages. On the T2-weighted FSE (A) only the right basal ganglia haemorrhage is visible as a small low signal intensity area. This haemorrhage causes much more susceptibility artefact on the T2*-

weighted GRE sequence (B), which reveals many additional central and peripheral foci of low signal intensity in both hemispheres, consistent with cerebral microhaemorrhages. 
conditions in the sense that it decreases with increasing histopathological changes.

\section{Applications \\ Research}

\section{- Schizophrenia}

In a group of subjects with chronic schizophrenia group mapping showed changes in MTR in both white and grey matter areas relative to a group of matched controls. ${ }^{26}$ These changes may reflect subtle changes involving neurones or neuronal processes, and are therefore an additional indication for the neuropathological background in schizophrenia.

\section{- Dementia}

MTI and regional MTR measurements have been studied in several subtypes of dementia. In vascular dementia, MTR of periventricular white matter lesions has been found to be lower in patients with Binswanger's disease than in patients with vascular disease but no dementia. ${ }^{27}$ Furthermore, MTR values correlate with neuropsychological measures, thereby suggesting that MTR reflects the severity of histopathological changes in vascular dementia.

MTR measurements of the hippocampus appear promising for the differentiation between $\mathrm{AD}$ and other types of dementia. ${ }^{27}$ Normal MTR values of the medial temporal lobe appear to be an early feature of $\mathrm{AD}^{27}$ and are already seen in patients with mild cognitive impairment. ${ }^{28}$ It was found that the MTR of grey matter was abnormally low both in patients with $\mathrm{AD}$ and patients with mild cognitive impairment, but MTR changes in the white matter were only present in patients with $\mathrm{AD}$, indicating a more advanced stage of the disease. $^{28}$ These findings suggest that MTR could be a novel MR measure for early diagnosis of $\mathrm{AD}$.

\section{- Trauma}

Traumatic brain injury may result in cerebral (cortical) contusions and/or diffuse axonal injury. The latter can cause subtle disruption of the structural integrity of brain tissue difficult to detect with conventional MRI. However, even in patients with only mild head injury abnormal MTR values have been found in the corpus callosum..$^{29}$ A decreased MTR has also been shown in white matter, which appeared normal on conventional MRI. ${ }^{30}$ The presence of abnormalities on MTI is often associated with persistent neurological deficits.

\section{- Tumours}

Experience with MT imaging in brain tumours is limited, but the significantly higher MTR of brain abscesses may help to differentiate them from cystic tumours. ${ }^{31}$ MTR can also distinguish between brain tumours which are hard and those that are soft on palpation during operation, the latter having lower MTR values. ${ }^{32}$

\section{- Multiple sclerosis}

In the past decade a number of studies have used MTI as a probe for tissue damage in MS. ${ }^{33}$ Evidence from correlative MTI/pathological studies suggests that the myelin content and the axonal count are the most relevant substrates of MTR changes in patients with MS. ${ }^{34} 35$

Hitherto, the use of MTI in MS has centred around three main fields: (a) its use in conjunction with the contrast agent gadolinium-DTPA ( $\mathrm{Gd}$ ) to improve the detection of new MS lesions, (b) assessment of MTR to distinguish lesions of different severity, and (c) assessment of changes in brain tissue that appears normal on conventional MRI. Due to their significance in MS research, we will focus on the latter two aspects.

Cross-sectional studies revealed a broad range of MTR values in MS lesions. However, it has consistently been shown that MS lesions returning a hypointense signal on Tlweighted MRI have a lower MTR than lesions which are Tlisointense. ${ }^{36}$

Correlation studies of standard T2-weighted MRI, T1weighted images with and without Gd enhancement, and MTR maps have revealed that the pathological substrate of even an apparently homogeneous group such as Gd enhancing lesions varies considerably. ${ }^{37}{ }^{38}$ Overall the MTR in lesions drops considerably when enhancement occurs, ${ }^{36}{ }^{37}$ but recovery of a lowered MTR may occur over the following months. ${ }^{38}$ Ring enhancing lesions, particularly their centre, display MTR values as low as in chronic Tl-hypointense lesions, ${ }^{36}{ }^{37}$ which result from severe demyelination and axonal loss, ${ }^{39}$ whereas homogeneously enhancing lesions have a significantly higher MTR. ${ }^{36}$

The severity of tissue damage-as reflected by changes in lesion MTR - has been shown to be modestly correlated with the course of MS. Patients with secondary progressive MS (SPMS) display lower lesion MTR than patients with benign $\mathrm{MS}^{40}$

A possible association between the MTR in brain tissue and the course of MS has also triggered a number of studies investigating tissue compartments appearing normal on conventional MRI. The MTR of the whole brain as well as of the segmented normal appearing white matter has consistently been shown to be lower in patients with MS than in healthy subjects. ${ }^{41-45}$

A number of studies have used MTI to investigate the time course of lesion development. They have shown that at least a subset of MS lesions develop against the background of tissue changes in the normal appearing white matter-the latter being a steady decrease of MTR in the respective regionoccurring up to 24 months before Gd enhancement becomes visible. ${ }^{46}{ }^{47}$ Lesions in regions showing a drop in MTR prior to Gd enhancement appear to be less likely to recover than lesions that did not. ${ }^{48}$ Although early recovery of lesions takes place as shown by the increase of an initially decreased lesion MTR during follow up, $^{38}$ an overall decrease of brain MTR occurs over time, whether assessed in lesions, normal appearing white matter, or the whole brain. ${ }^{33}{ }^{48-50}$ This has been demonstrated for a period of up to 4.5 years. ${ }^{50}$

Some MTR derived indices are associated with clinical disability. ${ }^{51}{ }^{52}$ A reduction of the whole brain MTR over one year has been shown to be an independent predictor of disability more than three years later ${ }^{40}$ and a low MTR in normal appearing white matter predicted disability over five years better than the MTR in lesions, suggesting that, in the long term, tissue pathology in normal appearing white matter may be more important than the lesions for the functional decline of patients with MS.

At least two factors exert significant influence on the associations between MTR derived indices and disability: the tissue compartment investigated and the MS subtype. In a study encompassing all subtypes of MS, the patients with SPMS had the lowest whole brain and lesion MTR values. ${ }^{53} \mathrm{~A}$ study including patients with primary progressive MS (PPMS), and patients with relapsing remitting (RR)-onset MS and disability, reported modest correlations of whole brain MTR with disability in both groups. ${ }^{54}$ However, in a study of patients with PPMS, MTR in the segmented grey matter onlybut not in normal appearing white matter-correlated with disability, suggesting that the grey matter may be particularly relevant for the development of disability in this subgroup. ${ }^{45}$

Recently, a novel quantitative MT technique allowing the estimation of the macromolecular proton fraction (f) and the 
bound pool $\mathrm{T} 2$ relaxation time (T2b) has been applied in patients with MS. Preliminary results showed significant differences for $\mathrm{f}$ and $\mathrm{T} 2 \mathrm{~b}$ between MS lesions, normal appearing white matter, and control white matter. This technique may achieve relative independence from the MT acquisition protocol while offering more pathologically specific information. ${ }^{55}$

After a decade of research and more than 200 studies into MT changes due to MS, guidelines for the implementation of MTR in large multicentre trials have been developed that should allow this technique to be used as a method to monitor treatment effects in patients with MS. ${ }^{56}$

\section{Clinical environment}

MTR is not firmly established as a clinical tool at present, which may be due to its restricted commercial availability. However, some of the research areas mentioned above may soon develop into clinical applications.

\section{Diffusion imaging}

Diffusion is often considered a functional technique. We argue that diffusion should be considered as a structural technique: diffusion indices such as mean diffusivity (MD) are measures of the cellular state, while diffusion anisotropy and tractography provide information about the structure of white matter.

\section{Principle}

MRI can be used to measure the self-diffusion of water-that is, the random motion of water molecules. If "diffusion encoding" gradients are applied, it can be shown that the NMR signal in the presence of diffusion experiences attenuation of amplitude due to the diffusion of water. Acquiring images with differing amounts of diffusion weighting (known as diffusion-weighted imaging, DWI) allows the diffusion coefficient to be measured. Diffusion in most brain tissues is restricted, so DWI measures the apparent diffusion coefficient (ADC). The most commonly used measure is $\mathrm{MD}$, where the diffusion coefficient is averaged over all directions. Diffusion in brain tissues can be isotropic, such as in CSF, in which the water can diffuse equally easily in each direction, or it can be anisotropic, such as in white matter tracts where diffusion is less restricted along the long axis of the white matter tract than it is perpendicular to the tract. The most commonly used index of diffusion anisotropy is probably fractional anisotropy, though there are various other indices.

Diffusion tensor imaging (DTI) allows the production of maps which show the principal direction of diffusion in any voxel. In structures with diffusion anisotropy, it has been shown that the principal direction of diffusion is parallel to the direction of the tract. Thus, tractography can be performed, where the path of these tracts can be traced through the brain. The tracts can be visualised by means of probability maps. This technique may be useful to investigate white matter changes in MS and other diseases of the central nervous system. ${ }^{57}$ At present, the effectiveness of tractography is limited by several factors, notably the low resolution of MRI compared with the size of most white matter tracts.

\section{Applications \\ Research}

\section{- Epilepsy}

Both decreases and increases in MD have been observed in patients with epilepsy scanned soon after a seizure. ${ }^{58}$ It has been suggested that the same mechanisms for diffusion change following acute stroke can occur in the ictal and postictal states. Changes in fractional anisotropy have been observed in patients with malformations of cortical development. ${ }^{59}$ These changes may partly be due to the high contrast between grey and white matter in the anisotropy maps, however, some changes observed on the fractional anisotropy maps have not been detected on the corresponding $\mathrm{Tl}$ weighted images.

\section{- Dementia}

A number of studies have explored the use of DWI in dementia, yielding at times contradictory results. Whereas some studies reported no significant regional differences between patients with mild cognitive impairment, probable $\mathrm{AD}$, and healthy subjects, ${ }^{60}$ others found a significant increase of the ADC in the hippocampal region in both mild cognitive impairment and $\mathrm{AD}$ compared with control subjects. ${ }^{61}$ Increased ADC values were also found in other brain regions including the temporal stem, posterior cingulate gyrus, corpus callosum, and parietal white matter. ${ }^{61}$

A selective involvement of white matter tracts has been found in patients with AD. DTI revealed a reduction in the integrity of association fibres such as the splenium of the corpus callosum, the superior longitudinal fasciculus, and the cingulum, while the integrity of the pyramidal tracts remained unchanged..$^{62}$ At $3 \mathrm{~T}$, fractional anisotropy has been found to be significantly reduced in the temporal white matter, posterior part of the corpus callosum, and anterior and posterior cingulated bundles in patients with $\mathrm{AD}^{63}$ Involvement of these white matter tracts correlated well with the degeneration of the cortical structures they are known to connect with, namely the posterior cerebral cortices and the hippocampus.

\section{- Trauma}

DWI is being used to assess the integrity of the brain tissue in the acute as well as in the subacute phase following head trauma. Decreased ADC values, reflecting restricted diffusion, have been found 1-18 days after head trauma, most frequently in the corpus callosum. ${ }^{64}$ The restricted diffusion could reflect cellular swelling (cytotoxic oedema), which persists into the subacute phase. Alternatively, the movement of water molecules could be impeded by the presence of fragmented membranes from ruptured axons. Conversely, repeated or longstanding head trauma can lead to an increase in ADC values, reflecting an increase in free water movement. Thus, histogram analysis of the whole brain in professional boxers revealed significantly higher average ADC values than in age matched normal controls. ${ }^{65}$ Furthermore, the average ADC correlated with the number of hospitalisations for boxing injuries, suggesting a cumulative effect of repeated blows to the head. Similarly, an increase in MD has also been shown several months after blunt head injury in patients who had unremarkable conventional MR images. ${ }^{66}$ The increase in $\mathrm{MD}$ extended to regions distant from the site of impact, suggesting an expansion of the extracellular space followed by neuronal or glial cell loss as a possible mechanism

\section{- Multiple sclerosis}

DW-MRI has been used over the past 10 years to probe MS. ${ }^{33}{ }^{67}$ A consistent finding has been an increased diffusivity compared with normal appearing white matter in MS lesions visible on T2-weighted MRI, either expressed by an elevated average $\mathrm{ADC}^{68}$ or $\mathrm{MD}^{69}$ Similarly fractional anisotropy has been shown to be lower in MS lesions than in normal appearing white matter. ${ }^{69} 70$

The degree to which changes in DWI occur appears to depend on the clinical course. Hence, MD has been found to 
be higher in lesions of patients with a secondary progressive course compared with those with RRMS ${ }^{71}$ or PPMS. ${ }^{72}$ The increased diffusivity in lesions of patients with SPMS corroborates the finding in SPMS of a higher proportion of hypointense lesions on Tl-weighted MRI ("black holes") which consistently display more abnormalities of diffusion than $\mathrm{Tl}$-isointense lesions ${ }^{73}{ }^{74}$ as long as they do not enhance after application of Gd; Gd enhancing Tl-hypointense lesions have been reported as having either different ${ }^{73}{ }^{74}$ or similar $^{75}$ diffusion properties compared with their non-enhancing counterparts. This may reflect a variable tissue composition of Gd enhancing lesions, depending on their age and severity.

Changes in the normal appearing brain tissue of patients with MS have also been detected by DWI. Such changes in water diffusion appear to occur very early in the course of MS and may precede the formation of new lesions. ${ }^{69}{ }^{76}$ The abnormalities of diffusion in normal appearing white matter appear to be more pronounced in periplaque normal appearing white matter than in remote regions ${ }^{77}$ and have been reported to be associated with the diffusivity in lesions ${ }^{78}$ as well as with the lesion load on conventional T2-weighted MRI. ${ }^{79} 80$

So far results regarding diffusion changes in patients with PPMS are conflicting. Evidence is there to suggest that in this subgroup of patients diffusion changes in the normal appearing white matter develop rather independently of lesions ${ }^{81}$ whereas other authors have detected an association between the extent of lesions and changes in the diffusivity of normal appearing white matter. ${ }^{80}$ The association of changes in diffusion of normal appearing white matter with disability in patients is still a matter of debate. Several recent studies, some of which used histogram analysis, reported such associations in patients with RRMS, SPMS, ${ }^{71}{ }^{82}$ and PPMS. $^{80}$

Some studies have focused on the grey matter of patients with MS by means of DWI. No changes were detected in the basal ganglia of MS patients ${ }^{83}$ or in the grey matter of RRMS patients ${ }^{84}$ whereas one study reported abnormalities of grey matter MD histograms in patients with PPMS or SPMS. ${ }^{85}$

\section{- Surgical applications}

It may be possible to use fractional anisotropy to better observe wallerian degeneration following surgical resection and thereby improve the assessment of postoperative sequelae, ${ }^{86}$ especially since a correlation of reduced fractional anisotropy with clinical deficit has been observed. ${ }^{87}$

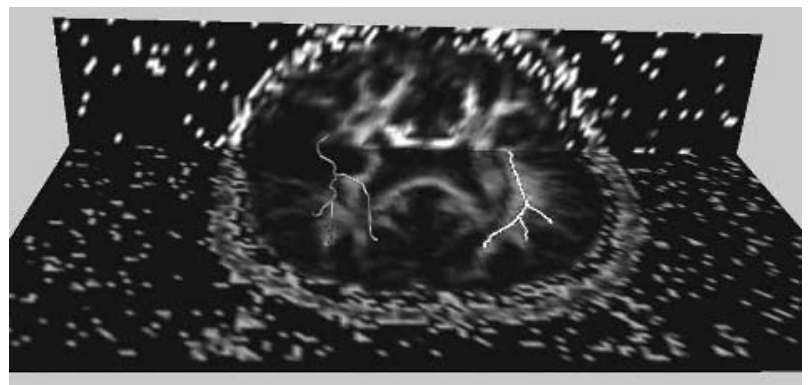

Figure 4 Composite three dimensional multisection representation of a diffusion fractional anisotropy image. Overlaid are two traces derived from tractography of the corresponding diffusion tensor data, showing the pathways taken by the superior longitudinal fasciculus (SLF) on each side. The SLF takes a normal path on the left (right side of image) but the right SLF (left side of image) takes a more circuitous route, because it has been displaced by a large tumour. Tractography here shows the preservation of the right SLF in spite of the tumour's presence, and also provides important additional information for any possible surgical intervention.
Furthermore, DTI and tractography have the potential to yield important information for preoperative management such as patient counselling and surgical planning. DTI can assess the effect of the tumour on the adjacent tracts by determining whether they are infiltrated or displaced; and tractography has the potential to trace the path of these tracts (fig 4). ${ }^{88}$ This information will enable both safer as well as more radical surgery.

\section{- Amyotrophic lateral sclerosis}

In subjects with amyotrophic lateral sclerosis, ADC and fractional anisotropy of the corticospinal tract correlate with disease duration and with disease severity, respectively. ${ }^{89}$ This opens the possibility of using these quantitative measures to monitor disease progression or possible drug effect in treatment trials.

\section{- Schizophrenia}

Conflicting results have been reported so far, possibly due to the varying methodologies used. A region of interest approach found increases in MD and decreases in fractional anisotropy in the splenium but not the genu of the corpus callosum. $^{90}$ Voxel-by-voxel approaches based on spatial normalisation and group mapping have either been negative ${ }^{91}$ or have reported widespread differences in fractional anisotropy and $\mathrm{MD}$, particularly in the prefrontal regions. ${ }^{92}$

\section{- Normal brain maturation and ageing}

From early life until adolescence ADC decreases and fractional anisotropy increases, ${ }^{93}$ whereas in the ageing brain ADC increases and fractional anisotropy decreases. ${ }^{94}$ These findings, however, have been reported in cross-sectional studies and are yet to be confirmed in longitudinal studies.

\section{Clinical environment}

\section{- Infarction}

DWI is far more sensitive for the detection of acute ischaemic changes than conventional MRI or CT. ${ }^{95}$ DWI has been shown to detect ischaemic lesions within minutes in experimental animal studies and within 30-90 minutes of an acute stroke in humans. ${ }^{96}$ Areas of acute ischaemia show restricted water diffusion and appear bright on DWI and dark on ADC maps. The precise mechanisms leading to a reduction in diffusion are still a matter of debate, but redistribution of extracellular water into the intracellular compartment (cytotoxic oedema) resulting in shrinkage of the extracellular space appears to be the most likely explanation.

Initially, changes on DWI were regarded as a marker of irreversible tissue damage and were used to define the ischaemic penumbra in combination with MR perfusion imaging (perfusion/diffusion mismatch). This assumption was subsequently proved to be erroneous, first by anecdotal reports of reversibility of DWI changes and later by quantitative ADC analysis, ${ }^{97}$ which demonstrated that tissue with ADC ratios of 0.9 (ADC reduced to $90 \%$ of the ADC of normal brain) and above was likely to recover whereas tissue with ADC ratios below that showed transition to infarction.

Appearances on DWI following stroke are time dependent: ADC values are low in the first week, become "pseudonormal" in the second week, and increase above the ADC of normal brain parenchyma thereafter. Decreased ADC values indicate with good sensitivity and specificity that an infarct is less than 10 days old.$^{98}$ MRI diffusion imaging can therefore be useful in identifying subacute infarcts. In the presence of several abnormalities suggestive of ischaemic stroke on 
T2-weighted images, DWI can help localise the acute lesion and determine its vascular territory, which may influence the clinical management. Acute ischaemia also causes a reduction of diffusion anisotropy. This effect is more marked in white matter than grey matter and fractional anisotropy may be a more sensitive measure of white matter ischaemia than DWI. ${ }^{99}$ DTI in the subacute phase can distinguish whether white matter tracts are distorted around the infarct or disrupted by it, with implications for functional recovery. ${ }^{100}$

DWI may also be useful to differentiate arterial infarcts from posterior reversible encephalopathy syndrome (PRES) and from venous infarcts. PRES is due to a breakthrough of the cerebral autoregulation and endothelial dysfunction, affecting predominately the posterior white matter with some involvement of the overlying cortex. The lesions are bright on $\mathrm{T} 2$-weighted images and may occasionally be difficult to distinguish from posterior circulation infarcts. However, on DWI these lesions are not hyperintense as acute arterial infarcts but appear isotense or hypointense, indicating the presence of vasogenic oedema, which is reversible. ${ }^{101}$ Venous infarcts initially cause vasogenic oedema with increased $\mathrm{ADC}$ values. At a later stage, the appearances are more complex and variable. There may be co-existence of vasogenic and cytotoxic oedema and the appearances may be further complicated by the presence of haematomas causing signal loss on DWI. ${ }^{102}$

\section{- Tumours}

While DWI has become an established tool in the clinical management of stroke, evidence is mounting of its usefulness in the clinical investigation of cerebral mass lesions. DWI of cerebral mass lesions is currently in transition from a research to a clinical application. We therefore decided to include this section among the clinical applications of diffusion imaging.

It must be emphasised that ADC measurements are essential for assessment of tumours, as the T2 shine-through effects may be predominant on DWI. Earlier studies ${ }^{103}$ established the inverse relationship between ADC values and histological cell count (tumour cellularity). Higher grade tumours, which have a higher density of cell nuclei, therefore have lower ADC values than low grade tumours. Statistically significant differences between the ADC values of high grade and low grade gliomas have been shown in a number of studies. ${ }^{103-105}$ Similarly, atypical or malignant (WHO grade 2 or 3) meningiomas have lower ADC values than benign meningiomas. ${ }^{106}$

It may be difficult to determine the degree of peritumoral neoplastic infiltration by gliomas on DWI alone ${ }^{104}$ but a recent study at $3 \mathrm{~T}$ demonstrated that DTI is capable of detecting subtle disruption of white matter tracts in high grade gliomas, consistent with tumour invasion. ${ }^{107}$ Primary central nervous system lymphomas tend to have lower ADC measurements than high grade gliomas, because they are highly cellular tumours. ${ }^{108}$

Of far greater importance is the potential to differentiate inflammatory lesions from tumours. The finding that lesions due to toxoplasmosis display significantly higher ADC values than lymphomas can be important for their differentiation in patients with AIDS. ${ }^{109}$ A similar problem can occur in the distinction between a purulent brain process and cystic brain tumours; a diagnosis that can be challenging on conventional MRI. Using DWI cerebral abscesses demonstrate a much greater restriction of diffusion than tumour cysts, resulting in significantly lower ADC values. ${ }^{110}$

Finally, arachnoid cysts and epidermoid tumours both have signal characteristics close to CSF on conventional MRI and are difficult to differentiate with this modality. This problem has now been definitively addressed by DWI, in which arachnoid cysts return a low signal, similar to CSF whereas epidermoid tumours return a high signal intensity, indicating restricted diffusion and thereby establishing the diagnosis.

\section{- Creutzfeldt-Jakob disease (CJD)}

DWI has become an essential tool in the diagnosis of CJD. The sporadic form shows areas of high signal intensity in the basal ganglia and cerebral cortex, which may precede changes on standard MRI, whereas the variant form is associated with high signal in the basal ganglia and mainly the pulvinar. Early changes become progressively hyperintense on DWI, unlike acute ischaemic infarcts, which tend to "pseudonormalise" within two weeks. The signal alteration corresponds histologically with areas of spongiform change and neuronal loss. ${ }^{11}$ DWI not only helps make an early diagnosis of CJD possible but has also been reported to have a high specificity and can differentiate between different forms of CJD. ${ }^{112}$

\section{THE NEXT TEN YEARS}

The continual development in MRI technology over the past two decades has produced both steady progress and dramatic breakthroughs. Given such a fertile field of invention, predictions of the future are difficult, but it can be hoped that there will be improvements in the hardware to further decrease imaging time and increase SNR, as well as enhancements in software for post-processing and data management.

\section{Acquisition development}

Conventional MRI uses one RF receiving coil to pick up the NMR signal from the body, and highly efficient configurations of transmitter coil (if there is a separate one), receiver coil, and RF receiver electronics have been used for many years in commercial MR scanners. A recent development, parallel imaging, ${ }^{113}$ combines an array of receiver coils in such a way that each coil oversamples the data required to reconstruct the MR image. The coils receive the signal simultaneously, resulting in effective time saving. Some SNR is lost in the process, but nevertheless, parallel imaging represents a new way of optimising MRI acquisition. Many commercial MR scanners are now available with parallel imaging ability, and as acquisition and reconstruction techniques improve, this may well become the modality of choice for many clinical MR examinations.

\section{High field imaging}

As the main magnetic field is increased, the amount of net magnetisation induced increases. From this consideration alone, it would seem advantageous to operate at the highest possible field and resonant frequency. Several obstacles to high field imaging are related to patients' safety and image quality.

\section{Patients' safety}

- RF penetration: As frequency increases, rather than passing into the centre of the body, the applied RF has a tendency to dissipate near the surface, causing adverse heating effects.

- Peripheral magnetic stimulation: If gradients are increased to overcome internal magnetic gradient fields, eventually the applied gradients start generating electrical currents in the patient causing tingling and even physical pain.

- Acoustic noise: As applied magnetic field and gradients increase, the motor effect means more acoustic noise is generated, which may become uncomfortable or even harmful for the patient. 


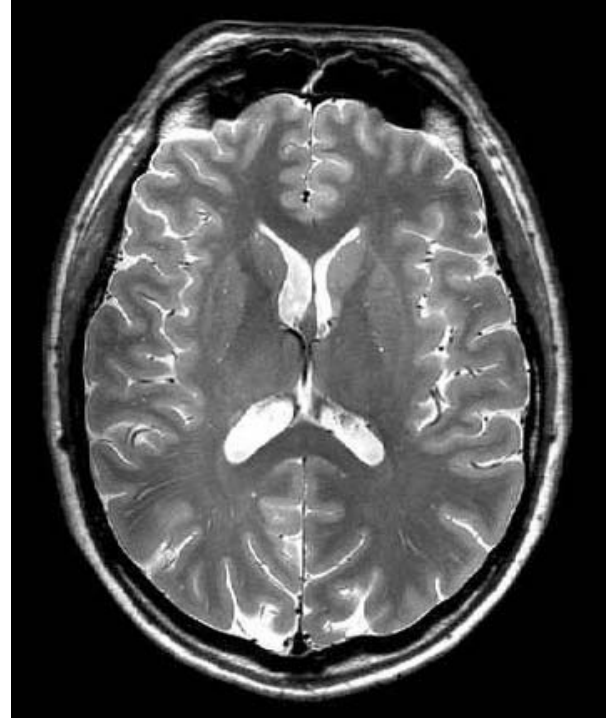

Figure 5 High resolution fast spin echo image, with a slice thickness of $2 \mathrm{~mm}$, and an in-plane resolution of $470 \times 470 \mu \mathrm{m}$ obtained at $4.7 \mathrm{~T}$. The signal to noise is very good, and allows visualisation of normally occurring Virchow-Robin spaces beyond the extent usually seen at 1.5 T imaging.

\section{Image degradation}

- RF homogeneity: Little RF reaches the centre of the body, so little MR signal is recovered from there.

- Main field inhomogeneity: As the applied magnetic field increases, internal magnetic gradient fields generated by differences between tissue and air cause larger image artefacts. These can be best overcome by larger field gradients.

As technology and MR engineering move on, so imaging at higher fields is becoming more feasible. Recent images obtained on prototype $4.7 \mathrm{~T}$ (fig 5$)^{114}$ and $8 \mathrm{~T}$ scanners bear this out. It is now possible to identify in vitro different types of cortex and even identify the corresponding cortical layers. ${ }^{115}$ It is hoped that this kind of resolution will be approached in vivo in the next decade. The current installation of $7 \mathrm{~T}$ and $11 \mathrm{~T}$ scanners may be opening new horizons in MRI and a first step in that direction.

\section{Post-processing and data management}

A 10 year old MR scanner can produce excellent structural MR images, but new MR scanners will soon be able to do much more. The modern MR scanner can be integrated with a picture archiving and communicating system (PACS), and further developments will lead to more integrated patient databases, image registration based acquisition prescription, comparison of newly acquired images with previous images of the same patient (serial comparisons) or against a group of similar subjects (group mapping techniques), and the automatic removal of extraneous image features (image segmentation to remove scalp, meninges etc). Given the power of computing available on new scanners, more advanced image processing techniques such as feature recognition may become possible. Interventional MRI, in which an MR scanner becomes truly interfaced with the surgical theatre, will also be developed further.

\section{CONCLUSION}

Structural MRI, using conventional T1, T2, and T2* contrasts, has become the accepted standard for routine examination of the brain, offering exquisite anatomical detail and high sensitivity to pathological changes. New quantitative measures of magnetisation transfer and diffusion are being widely used in research and some will soon become standard clinical tools. Future developments promise an increase in both sensitivity and specificity.

\section{ACKNOWLEDGEMENTS}

We thank Philippa Bartlett, Sofia Eriksson, Roger Ordidge, and Geoff Parker for providing some of the figures.

\section{Authors' affiliations}

M Symms, Department of Clinical and Experimental Epilepsy, Institute of Neurology, London, UK

H R Jäger, T A Yousry, Lysholm Department of Neuroradiology,

National Hospital of Neurology and Neurosurgery, Institute of Neurology, London, UK

K Schmierer, Department of Neuroinflammation, NMR Research Unit, Institute of Neurology, London, UK; and Klinik für Neurologie, Chasité, Humbeldt Universitãt zu Berlin, Berlin, Germany

Competing interests: none declared

\section{REFERENCES}

1 Bloch F. Nuclear Induction. Phys Rev 1946;70:460-74.

2 Purcell EM, Torrey HC, Pound RV. Resonance absorption by nuclear magnetic moments in a solid. Phys Rev 1946;69:37-8.

3 Mansfield P, Grannell PK. NMR "diffraction" in solids? J Phys C: Solid State Phys 1973;6:L422-L426.

4 Lauterbur P. Image formation by local induced interactions: examples employing nuclear magnetic resonance. Nature 1973;242:190-1.

5 Moran PR. A flow velocity zeugmatographic interlace for NMR imaging in humans. Magn Reson Imaging 1982;1:197-203.

6 Jezzard P, Matthews PM, Smith SM. Functional MRI: an introduction to methods. Oxford: Oxford University Press, 2001.

7 McRobbie DW, Moore EA, Graves MJ, et al. MRI: From Picture to Proton. Cambridge: Cambridge University Press, 2003.

8 Tofts PS. Quantitative MRI of the Brain: Measuring Changes Caused by Disease. Chichester: Wiley, 2003.

9 Fox NC, Freeborough PA. Brain atrophy progression measured from registered serial MRI: validation and application to Alzheimer's disease. $J$ Magn Reson Imaging 1997;7:1069-75.

10 Yousry I, Camelio S, Wiesmann M, et al. Detailed magnetic resonance imaging anatomy of the cisternal segment of the abducent nerve: Dorello's canal and neurovascular relationships and landmarks. J Neurosurg 1999;91:276-83.

11 Yousry I, Moriggl B, Dieterich M, et al. MR anatomy of the proximal cisternal segment of the trochlear nerve: neurovascular relationships and landmarks. Radiology 2002;223:31-8.

12 Yousry I, Dieterich M, Naidich TP, et al. Superior oblique myokymia magnetic resonance imaging support for the neurovascular compression hypothesis. Ann Neurol 2002;51:361-8.

13 Jackson GD, Connelly A, Duncan JS, et al. Detection of hippocampal pathology in intractable partial epilepsy: increased sensitivity with quantitative magnetic resonance T2 relaxometry. Neurology 1993:43: 1793-9

14 Graham JM, Paley MNJ, Grunewald, RA, et al. Brain iron deposition in Parkinson's disease imaged using the PRIME magnetic resonance sequence. Brain 2000;123:2423-31.

15 Perl J 2nd, Tkach JA, Porras-Jimenez M, et al. Hemorrhage detected using $M R$ imaging in the setting of acute stroke: an in vivo model. AJNR Am J Neuroradiol 1999;20:1863-70.

16 Lin DD, Filippi CG, Steever AB, et al. Detection of intracranial hemorrhage: comparison between gradient-echo images and $b(0)$ images obtained from diffusion-weighted echo-planar sequences. AJNR Am J Neuroradiol 2001;22:1275-81

17 Mitchell P, Wilkinson ID, Hoggard N, et al. Detection of subarachnoid haemorrhage with magnetic resonance imaging. J Neurol Neurosurg Psychiatry 2001;70:205-11.

18 Fazekas F, Kleinert R, Roob G, et al. Histopathologic analysis of foci of signal loss on gradient-echo T2*-weighted MR images in patients with spontaneous intracerebral hemorrhage: evidence of microangiography-related microbleeds. AJNR Am J Neuroradiol 1999;20:637-42.

19 Greenberg SM, O'Donnell HC, Schaefer PW, et al. MRI detection of new hemorrhages: potential marker of progression in cerebral amyloid angiopathy. Neurology 1999;53:1135-8.

20 Dichgans $M$, Holtmannspotter $M$, Herzog J, et al. Cerebral microbleeds in CADASIL: a gradient-echo magnetic resonance imaging and autopsy study. Stroke 2002;33:67-71

21 Roob G, Schmidt R, Kapeller P, et al. MRI evidence of past cerebral microbleeds in a healthy elderly population. Neurology 1999;52:991-4.

22 Tsushima Y, Aoki J, Endo K. Brain microhemorrhages detected on T2*weighted gradient-echo MR images. AJNR Am J Neuroradiol 2003;24:88-96 
23 Scheid R, Preul C, Gruber O, et al. Diffuse axonal injury associated with chronic traumatic brain injury: evidence from T2*-weighted gradient-echo imaging at 3 T. AJNR Am J Neuroradiol 2003;24:1049-56.

24 Parker GJM, Barker GJ, Tofts PS. Accurate multislice gradient echo T1 measurement in the presence of non-ideal RF pulse shape and RF field nonuniformity. Magn Reson Med 2001;45:838-45.

25 Stevenson VL, Parker GJM, Barker GJ, et al. Variations in T1 and T2 relaxation times of normal appearing white matter and lesions in multiple sclerosis. J Neurol Sci 2000;178:81-7.

26 Foong J, Symms MR, Barker GJ, et al. Investigating regional white matter in schizophrenia using diffusion tensor imaging. NeuroReport 2002;13:333-6.

27 Hanyu H, Asano T, Iwamoto T, et al. Magnetization transfer measurements of the hippocampus in patients with Alzheimer's disease, vascular dementia, and other types of dementia. AJNR Am J Neuroradiol 2000;21:1235-42.

28 Kabani NJ, Sled JG, Chertkow H. Magnetization transfer ratio in mild cognitive impairment and dementia of Alzheimer's type. Neuroimage 2002;15:604-10.

29 McGowan JC, Yang JH, Plotkin RC, et al. Magnetization transfer imaging in the detection of injury associated with mild head trauma. AJNR Am J Neuroradiol 2000;21:875-80.

30 Sinson G, Bagley $\sqcup$, Cecil KM, et al. Magnetization transfer imaging and proton MR spectroscopy in the evaluation of axonal injury: correlation with clinical outcome after traumatic brain injury. Am J Neuroradiol 2001 22:143-51.

31 Pui MH. Magnetization transfer analysis of brain tumor, infection, and infarction. J Magn Reson Imaging 2000;12:395-9.

32 Okumura A, Takenaka K, Nishimura Y, et al. The characterization of human brain tumor using magnetization transfer technique in magnetic resonance imaging. Neurol Res 1999;21:250-4.

33 Filippi M, Rocca MA, Comi G. The use of quantitative magnetic-resonancebased techniques to monitor the evolution of multiple sclerosis. Lancet Neurol 2003;2:337-46.

34 van Waesberghe JH, Kamphorst W, De Groot CJ, et al. Axonal loss in multiple sclerosis lesions: magnetic resonance imaging insights into substrates of disability. Ann Neurol 1999;46:747-54.

35 Mottershead JP, Schmierer K, Clemence M, et al. High field MRI correlates of myelin content and axonal density in multiple sclerosis: a post-mortem study of the spinal cord. J Neurol 2003;250:1293-301.

36 Petrella JR, Grossman RI, McGowan JC, et al. Multiple sclerosis lesions: relationship between MR enhancement pattern and magnetization transfer effect. AJNR Am J Neuroradiol 1996;17:1041-9.

37 Rovira A, Alonso J, Cucurella G, et al. Evolution of multiple sclerosis lesions on serial contrast-enhanced T1-weighted and magnetization-transfer MR images. AJNR Am J Neuroradiol 1999;20:1939-45.

38 Lai HM, Davie CA, Gass A, et al. Serial magnetisation transfer ratios in gadolinium-enhancing lesions in multiple sclerosis. J Neurol 1997;244:308-11

39 van Walderveen MA, Kamphorst W, Scheltens P, et al. Histopathologic correlate of hypointense lesions on T1-weighted spin-echo MRI in multiple sclerosis. Neurology 1998;50:1282-8.

40 Davie CA, Silver NC, Barker GJ, et al. Does the extent of axonal loss and demyelination from chronic lesions in multiple sclerosis correlate with the clinical subgroup? J Neurol Neurosurg Psychiatry 1999;67:710-15.

41 van Buchem MA, McGowan JC, Kolson DL, et al. Quantitative volumetric magnetization transfer analysis in multiple sclerosis: estimation of macroscopic and microscopic disease burden. Magn Reson Med 1996;36:632-6.

42 Cercignani $M$, Bozzali $M$, lannucci $G$, et al. Magnetisation transfer ratio and mean diffusivity of normal appearing white and grey matter from patients with multiple sclerosis. J Neurol Neurosurg Psychiatry 2001;70:311-17.

43 Griffin CM, Chard DT, Parker GJ, et al. The relationship between lesion and normal appearing brain tissue abnormalities in early relapsing remitting multiple sclerosis. J Neurol 2002;249:193-9.

44 De Stefano N, Narayanan S, Francis SJ, et al. Diffuse axonal and tissue injury in patients with multiple sclerosis with low cerebral lesion load and no disability. Arch Neurol 2002;59:1565-71.

45 Dehmeshki J, Chard DT, Leary SM, et al. The normal appearing grey matter in primary progressive multiple sclerosis: a magnetisation transfer imaging study. J Neurol 2003;250:67-74.

46 Goodkin DE, Rooney WD, Sloan R, et al. A serial study of new MS lesions and the white matter from which they arise. Neurology 1998;51:1689-97.

47 Filippi M, Rocca MA, Martino G, et al. Magnetization transfer changes in the normal appearing white matter precede the appearance of enhancing lesions in patients with multiple sclerosis. Ann Neurol 1998:43:809-14.

48 Laule C, Vavasour IM, Whittall KP, et al. Evolution of focal and diffuse magnetisation transfer abnormalities in multiple sclerosis. J Neurol 2003;250:924-31.

49 van Waesberghe JH, van Walderveen MA, Castelijns JA, et al. Patterns of lesion development in multiple sclerosis: longitudinal observations with T1weighted spin-echo and magnetization transfer MR. AJNR Am J Neuroradiol 1998; 19:675-83.

50 Rovaris M, Agosta F, Sormani MP, et al. Conventional and magnetization transfer MRI predictors of clinical multiple sclerosis evolution: a medium-term follow-up study. Brain 2003;126:2323-32.

51 Gass A, Barker GJ, Kidd D, et al. Correlation of magnetization transfer ratio with clinical disability in multiple sclerosis. Ann Neurol 1994;36:62-7.

52 Udupa JK, Nyul LG, Ge Y, et al. Multiprotocol MR image segmentation in multiple sclerosis: experience with over 1000 studies. Acad Radiol 2001;8:1116-26.
53 Filippi $M$, lannucci $G$, Tortorella $C$, et al. Comparison of MS clinical phenotypes using conventional and magnetization transfer MRI. Neurology 1999;52:588-94.

54 Dehmeshki J, Silver NC, Leary SM, et al. Magnetisation transfer ratio histogram analysis of primary progressive and other multiple sclerosis subgroups. J Neurol Sci 2001;185:11-17.

55 Davies GR, Ramani A, Dalton CM, et al. Preliminary magnetic resonance study of the macromolecular proton fraction in white matter: a potential marker of myelin? Mult Scler 2003;9:246-9.

56 Horsfield MA, Barker GJ, Barkhof F, et al. Guidelines for using quantitative magnetization transfer magnetic resonance imaging for monitoring treatment of multiple sclerosis. J Magn Reson Imaging 2003;17:389-97.

57 Ciccarelli O, Toosy AT, Parker GJ, et al. Diffusion tractography based group mapping of major white-matter pathways in the human brain. Neuroimage 2003;19:1545-55.

58 Wieshmann UC, Symms MR, Shorvon SD. Diffusion changes in status epilepticus. Lancet 1997;350:493-4.

59 Eriksson SH, Rugg-Gunn EJ, Symms MR, et al. Diffusion tensor imaging in patients with epilepsy and malformations of cortical development. Brain 2001; 124:617-26.

60 Bozzao A, Floris R, Baviera ME, et al. Diffusion and perfusion MR imaging in cases of Alzheimer's disease: correlations with cortical atrophy and lesion load. AJNR Am J Neuroradiol 2001;22:1030-6.

61 Kantarci K, Jack CR Jr, Xu YC, et al. Mild cognitive impairment and Alzheimer disease: regional diffusivity of water. Radiology 2001;219:101-7.

62 Rose SE, Chen F, Chalk JB, et al. Loss of connectivity in Alzheimer's disease: an evaluation of white matter tract integrity with colour coded MR diffusion tensor imaging. J Neurol Neurosurg Psychiatry 2000;69:528-30.

63 Takahashi S, Yonezawa H, Takahashi J, et al. Selective reduction of diffusion anisotropy in white matter of Alzheimer disease brains measured by 3.0 Tesla magnetic resonance imaging. Neurosci Lett 2002;332:45-8.

64 Liu AY, Maldjian JA, Bagley U, et al. Traumatic brain injury; diffusionweighted MR imaging finding. AJNR Am J Neuroradiol 1999;20:1636-41.

65 Zhang L, Ravdin LD, Relkin N, et al. Increased diffusion in the brain of professional boxers: a preclinical sign of traumatic brain injury? AJNR Am J Neuroradiol 2003;24:52-7.

66 Rugg-Gunn FJ, Symms MR, Barker GJ, et al. Diffusion imaging shows abnormalities after blunt head trauma when conventional magnetic resonance imaging is normal. J Neurol Neurosurg Psychiatry 2001;70:530-3

67 Horsfield MA, Jones DK. Applications of diffusion-weighted and diffusion tensor MRI to white matter diseases-a review. NMR Biomed 2002; 15:570-7

68 Christiansen P, Gideon P, Thomsen C, et al. Increased water self-diffusion in chronic plaques and in apparently normal white matter in patients with multiple sclerosis. Acta Neurol Scand 1993;87:195-9.

69 Werring DJ, Brassat D, Droogan AG, et al. The pathogenesis of lesions and normal-appearing white matter changes in multiple sclerosis: a serial diffusion MRI study. Brain 2000;123:1667-76.

70 Castriota-Scanderbeg A, Fasano F, Hagberg G, et al. Coefficient D (av) is move sensitive than fractional anisotropy in monitoring progression of irreversible tissue damage in total nonactive multiple sclerosis lesions. AJNR Am J Neuroradiol 2003;24:663-70.

71 Castriota-Scanderbeg A, Tomaiuolo F, Sabatini U, et al. Demyelinating plaques in relapsing-remitting and secondary-progressive multiple sclerosis: assessment with diffusion MR imaging. AJNR Am J Neuroradiol 2000;21:862-8

72 Rovaris M, Bozzali M, lannucci G, et al. Assessment of normal-appearing white and gray matter in patients with primary progressive multiple sclerosis: a diffusion-tensor magnetic resonance imaging study. Arch Neurol 2002;59:1406-12.

73 Nusbaum AO, Lu D, Tang CY, et al. Quantitative diffusion measurements in focal multiple sclerosis lesions: correlations with appearance on Tl-weighted MR images. AJR Am J Roentgenol 2000;175:821-5.

74 Roychowdhury S, Maldjian JA, Grossman RI. Multiple sclerosis: comparison of trace apparent diffusion coefficients with MR enhancement pattern of lesions. AJNR Am J Neuroradiol 2000;21:869-74.

75 Filippi $M$, lannucci $G$, Cercignani $M$, et al. A quantitative study of water diffusion in multiple sclerosis lesions and normal-appearing white matter using echo-planar imaging. Arch Neurol 2000;57:1017-21.

76 Rocca MA, Cercignani M, lannucci G, et al. Weekly diffusion-weighted imaging of normal-appearing white matter in MS. Neurology 2000;55:882-4.

77 Guo AC, Jewells VL, Provenzale JM. Analysis of normal-appearing white matter in multiple sclerosis: comparison of diffusion tensor MR imaging and magnetization transfer imaging. AJNR Am J Neuroradiol 2001;22:1893-900.

78 Werring DJ, Clark CA, Droogan AG, et al. Water diffusion is elevated in widespread regions of normal-appearing white matter in multiple sclerosis and correlates with diffusion in focal lesions. Mult Scler 2001;7:83-9

79 Iannucci G, Rovaris M, Giacomotti L, et al. Correlation of multiple sclerosis measures derived from $\mathrm{T} 2$-weighted, $\mathrm{T} 1$-weighted, magnetization transfer, and diffusion tensor MR imaging. AJNR Am J Neuroradiol 2001;22:1462-7.

80 Schmierer K, Altmann DR, Kassim N, et al. Progressive change in primary progressive MS normal-appearing white matter: a serial diffusion magnetic resonance imaging study. Mult Scler 2004:10:182-7.

81 Rocca MA, lannucci $G$, Rovaris $M$, et al. Occult tissue damage in patients with primary progressive multiple sclerosis is independent of T2-visible lesions - a diffusion tensor MR study. J Neurol 2003;250:456-60. 
82 Cercignani $M$, Inglese $M$, Pagani $E$, et al. Mean diffusivity and fractional anisotropy histograms of patients with multiple sclerosis. AJNR Am J Neuroradiol 2001;22:952-8.

83 Holtmannspotter $M$, Inglese $M$, Rovaris $M$, et al. A diffusion tensor MRI study of basal ganglia from patients with ADEM. J Neurol Sci 2003;206:27-30.

84 Griffin CM, Chard DT, Ciccarelli O, et al. Diffusion tensor imaging in early relapsing-remitting multiple sclerosis. Mult Scler 2001;7:290-7.

85 Bozzali M, Cercignani M, Sormani MP, et al. Quantification of brain gray matter damage in different MS phenotypes by use of diffusion tensor MR imaging. AJNR Am J Neuroradiol 2002;23:985-8.

86 Pierpaoli C, Barnett A, Pajevic S, et al. Water diffusion changes in Wallerian degeneration and their dependence on white matter architecture. Neuroimage 2001;13:1174-85.

87 Wieshmann UC, Symms MR, Clark CA, et al. Wallerian degeneration in the optic radiation after temporal lobectomy demonstrated in vivo with diffusion tensor imaging. Epilepsia 1999;40:1155-8.

88 Clark CA, Barrick TR, Murphy MM, Bell BA. White matter fiber tracking in patients with space-occupying lesions of the brain: a new technique for neurosurgical planning? Neuroimage 2003;20:1601-8.

89 Ellis CM, Simmons A, Jones DK, et al. Diffusion tensor MRI assesses corticospinal tract damage in ALS. Neurology 1999;53:1051-8.

90 Foong J, Maier M, Clark C, et al. Neuropathological abnormalities of the corpus callosum in schizophrenia: a diffusion tensor imaging study. J Neurol Neurosurg Psychiatry 2000;68:242-4.

91 Foong J, Symms MR, Barker GJ, et al. Investigating regional white matter in schizophrenia using diffusion tensor imaging. NeuroReport 2002;13:333-6.

92 Buchsbaum MS, Tang CY, Peled S, et al. MRI white matter diffusion anisotropy and PET metabolic rate in schizophrenia. NeuroReport 1998:9:425-30

93 Mukheriee $\mathbf{P}$, Miller JH, Shimony JS, et al. Normal brain maturation during childhood: developmental trends characterized with diffusion-tensor MR imaging. Radiology 2001;221:349-58.

94 Pfefferbaum A, Sullivan EV, Hedehus $M$, et al. Age-related decline in brain white matter anisotropy measured with spatially corrected echo-planar diffusion tensor imaging. Magn Reson Med 2000;44:259-68.

95 Mullins ME, Schaefer PW, Sorensen AG, et al. CT and conventional and diffusion-weighted MR imaging in acute stroke: study in 691 patients at presentation to the emergency department. Radiology 2002;224:353-60.

96 Mullins ME, Schaefer PW, Sorensen AG, et al. Magnetic resonance imaging of cerebral infarction. Top Magn Reson Imaging 1998;9:199-207.

97 Desmond PM, Lovell C, Rawlinson A, et al. The value of apparent diffusion coefficient maps in early cerebral ischemia. AJNR Am J Neuroradiol 2001;22:1260-7.

98 Lansberg MG, Thiijs VN, O'Brien MW, et al. Evolution of apparent diffusion coefficient, diffusion-weighted, and T2-weighted signal intensity of acute stroke. AJNR Am J Neuroradiol 2001;22:637.
99 Mukheriee $\mathbf{P}$, Bahn MM, McKinstry, et al. Differences between gray matter and white matter water diffusion in stroke; diffusion- tensor MR imaging in 12 patients. Neuroradiol 2000;215:211-20.

100 Gillard JH, Papadakis NG, Martin K, et al. MR diffusion tensor imaging of white matter tract disruption in stroke at 3T. Br J Radiol 2001;74:642-7.

101 Lamy C, Oppenheim C, Meder JF, et al. Neuroimaging in posterior reversible encephalopathy syndrome. J Neuroimaging 2004;14:89-96.

102 Lovblad KO, Bassetti C, Schneider J, et al. Diffusion-weighted MR in cerebral venous thrombosis. Cerebrovasc Dis 2001:11:169-76.

103 Sugahara T, Korogi Y, Kochi M, et al. Usefulness of diffusion-weighted MRI with echo-planar technique in the evaluation of cellularity in gliomas. J Magn Reson Imaging 1999;9:53-60.

104 Kono K, Inove Y, Nakayama K, et al. The role of diffusion-weighted imaging in patients with brain tumors. AJNR Am J Neuroradiol 2001;22:1081-8.

105 Yang D, Korogi T, Sugahara T, et al. Cerebral gliomas: prospective comparison of multivoxel $2 \mathrm{D}$ chemical- shift imaging proton $M R$ spectroscopy, echoplanar perfusion and diffusion-weighted MRI. Neuroradiol 2002;44:656-66.

106 Filippi CG, Edgar MA, Ulug AM, et al. Appearance of meningiomas on diffusion-weighted images: correlating diffusion constants with histopathologic findings. AJNR Am J Neuroradiol 2001;22:65-72.

107 Price SJ, Burnet NG, Donovan T, et al. Diffusion tensor imaging of brain tumours at $3 \mathrm{~T}$ : a potential tool for assessing white matter tract invasion? Clin Radiol 2003;58:455-62.

108 Guo AC, Cummings TJ, Dash RC, et al. Lymphomas and high-grade astrocytomas: comparison of water diffusibility and histologic characteristics. Radiology 2002;224:177-83.

109 Camacho DL, Smith JK, Castillo M. Differentiation of toxoplasmosis and lymphoma in AIDS patients by using apparent diffusion coefficients AJNR Am J Neuroradiol 2003;24:633-7.

110 Guzman R, Barth A, Lovblad KO, et al. Use of diffusion-weighted magnetic resonance imaging in differentiating purulent brain processes from cystic brain tumors. J Neurosurg 2002;97:1 101-7.

111 Mittal S, Farmer P, Kalina P, et al. Correlation of diffusion-weighted magnetic resonance imaging with neuropathology in Creutzfeldt-Jakob disease. Arch Neurol 2002;59:128-34.

112 Demaerel P, Sciot R, Robberecht W, et al. Accuracy of diffusion-weighted MR imaging in the diagnosis of sporadic Creutzfeldt-Jakob disease. J Neurol 2003;250:222-5.

113 Pruessmann KP, Weiger M, Scheidegger MB, et al. SENSE: Sensitivity Encoding for Fast MRI. Magn Reson Med 1999;42:952-62.

114 De Vita E, Thomas DL, Roberts S, et al. High resolution MRI of the brain at 4.7 Tesla using fast spin echo imaging. Br J Radiol 2003;76:631-7.

115 Fatterpekar GM, Naidich TP, Delman BN, et al. Cytoarchitecture of the human cerebral cortex: MR microscopy of excised specimens at 9. 4 Tesla AJNR Am J Neuroradiol, 2002;23:1313-21. 